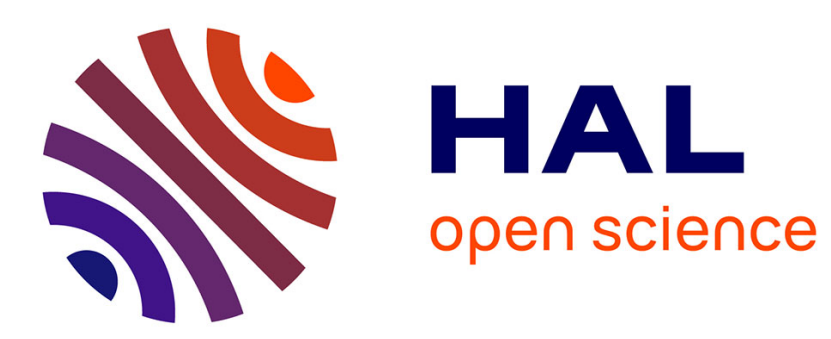

\title{
Radiative heat transfer at nanoscale mediated by surface plasmons for highly doped silicon.
}

Emmanuel Rousseau, Marine Laroche, Jean-Jacques Greffet

\section{To cite this version:}

Emmanuel Rousseau, Marine Laroche, Jean-Jacques Greffet. Radiative heat transfer at nanoscale mediated by surface plasmons for highly doped silicon.. Applied Physics Letters, 2009, 95, pp.231913. 10.1063/1.3271681 . hal-00428587

\section{HAL Id: hal-00428587 \\ https://hal.science/hal-00428587}

Submitted on 29 Oct 2009

HAL is a multi-disciplinary open access archive for the deposit and dissemination of scientific research documents, whether they are published or not. The documents may come from teaching and research institutions in France or abroad, or from public or private research centers.
L'archive ouverte pluridisciplinaire HAL, est destinée au dépôt et à la diffusion de documents scientifiques de niveau recherche, publiés ou non, émanant des établissements d'enseignement et de recherche français ou étrangers, des laboratoires publics ou privés. 


\title{
Radiative heat transfer at nanoscale mediated by surface plasmons for highly doped silicon.
}

\author{
Emmanuel Rousseau *, Marine Laroche, and Jean-Jacques Greffet \\ Laboratoire Charles Fabry \\ Institut d'Optique, CNRS, Université Paris-sud \\ 2 av Fresnel \\ 91127 Palaiseau cedex, France
}

\begin{abstract}
In this letter, we revisit the role of surface plasmons for nanoscale radiative heat transfer between doped silicon surfaces. We derive a new accurate and closed-form expression of the radiative nearfield heat transfer. We also analyse the flux and find that there is a doping level that maximizes the heat flux.
\end{abstract}

Radiative heat transfer at nanoscale can be orders of magnitude larger than predicted by Stefan's law. This is now well-known and well documented ${ }^{1-12}$. Several groups have reported experimental evidence of the effect of the enhancement of the flux ${ }^{13,14}$. More recently, the effect has been investigated in the nanometer regime $^{15-17}$. Finally, experimental measurements with a well-controlled geometry in the nanometer regime have confirmed the theoretical predictions ${ }^{18,19}$. These experiments have been performed for silica taking adavantage of the strong contribution of surface phonon polaritons to the nanoscale radiative heat transfer predicted in ref. ${ }^{11,12}$. In this paper, we discuss the contribution of surface plasmons to the nanoscale radiative heat transfer. It is well-known that surface plasmons do not play any role in the heat transfer between two parallel metallic surfaces because they cannot be excited thermally. Indeed, typical energies of surface plasmons are larger than $2 \mathrm{eV}$. However, when dealing with highly doped silicon, the surface plasmon frequency is in the infrared. This effect was extensively analysed by $\mathrm{Fu}$ and $\mathrm{Zhang}^{20}$. In this paper, we report a detailed analysis of the contribution of the surface plasmon to the nanoscale heat transfer. We derive an accurate asymptotic closed-form expression of this contribution. Finally, we show that the flux can be maximized by properly chosing the doping. The heat transfer coefficient is then found to be on the same order of magnitude than for silica and much larger than for metals. The system considered in this letter consists of a gap separating two homogeneous half spaces. Doped silicon is characterized by its local isotropic dielectric constant. It includes an intrinsic contribution denoted $\varepsilon_{\text {int }}$ and the carrier contribution which can be either electrons $(e)$ or holes $(h)$. The intrinsic contribution is found from optical data at room temperature ${ }^{21}$. The carrier contribution is given by a Drude model ${ }^{20}$.

The plates temperatures are supposed to be uniform and noted $T$ and $T+\delta T$. We will assume that $\delta T / T<<$ 1 so that we can introduce a heat transfer coefficient $\varphi(d, T)=h(d, T) \delta T$ where $\varphi(d, T)$ is the radiative flux

*Electronic mail: emmanuel.rousseau@institutoptique.fr per unit area. The radiative heat transfer coefficient can be cast in the form ${ }^{3,7-9,11}$ :

$$
h(d, T)=\sum_{j} \int_{0}^{\infty} d u\left[h_{j}^{\text {prop }}(u, T, d)+h_{j}^{\text {evan }}(u, T, d)\right]
$$

where $u=\hbar \omega / k_{B} T$, prop and evan stand respectively for propagating and evanescent waves and $j=s, p$ stands for the polarization. The coefficients are given by:

$$
\begin{array}{r}
h_{j}^{\text {prop }}(u, d, T)=h_{0}(u) \int_{0}^{\omega / c} \kappa d \kappa\left(\frac{c}{\omega}\right)^{2} \times \\
\frac{\left(1-\left|r_{31}^{j}\right|^{2}\right)\left(1-\left|r_{32}^{j}\right|^{2}\right)}{\left|1-r_{31}^{j} r_{32}^{j} e^{2 i \gamma d}\right|^{2}} \\
h_{j}^{\text {evan }}(u, d, T)=h_{0}(u) \int_{\omega / c}^{\infty} \kappa d \kappa\left(\frac{c}{\omega}\right)^{2} \times \\
\frac{4 \operatorname{Im}\left(r_{31}^{j}\right) \operatorname{Im}\left(r_{32}^{j}\right)}{\left|1-r_{31}^{j} r_{32}^{j} e^{-2 \operatorname{Im}[\gamma] d}\right|^{2}} e^{-2 \operatorname{Im}[\gamma] d}
\end{array}
$$

$h_{0}(u)$ is the derivative of the Planck function: $h_{0}(u)=$ $\frac{\partial L}{\partial T}(u)=\frac{3}{2 \pi^{2}} \frac{g_{0}}{\lambda_{T}^{2}} u^{4} \frac{e^{u}}{\left(e^{u}-1\right)^{2}}$ with $g_{0}=\pi^{2} k_{b}^{2} T / 3 h$ the quantum of thermal conductance and $\lambda_{T}=\hbar c / k_{b} T$ the thermal wavelength. The Fresnel coefficients are given by : $r_{3 m}^{s}=\frac{\gamma-\gamma_{m}}{\gamma+\gamma_{m}}$ and $r_{3 m}^{p}=\frac{\varepsilon_{m}(\omega) \gamma-\gamma_{m}}{\varepsilon_{m}(\omega) \gamma+\gamma_{m}}$, where 3 denotes the vacuum and $m$ stands for one of the two plates $m=1,2$. $p$ means Transverse Magnetic $(T M)$ polarization and $s$ Transverse Electric $(T E) . \gamma$ is the normal component of the wave vector in the gap and $\gamma_{m}$ is the wave vector in the media defined as $\gamma_{m}=\sqrt{\varepsilon_{m}(\omega)(\omega / c)^{2}-\kappa^{2}}$. To compute the total flux, it is necessary to add the contributions of all the modes by integrating over $\kappa$ and $\omega$.

As an example, we plot in Fig.1 the heat transfer coefficient versus the distance for a doping level equal to $N_{e}=2.10^{19} \mathrm{~cm}^{-3}$. Two features appear clearly. It is seen that the contribution of s-polarized evanescent waves first increases and then saturates as the gap width decreases. On the other hand, the contribution of the ppolarized evanescent waves varies as $d^{-2}$ and dominates for gaps smaller than $50 \mathrm{~nm}$. This contribution does not exist for intrinsic silicon. It can be attributed to the contribution of the surface plasmon polariton associated 


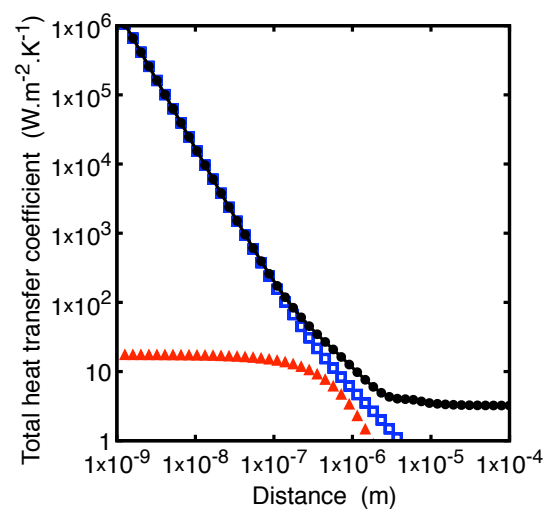

FIG. 1: Colors are available on-line. Total heat transfer coefficient at high doping level: $N_{e}=2.10^{19} \mathrm{~cm}^{-3}$. Dot black curve: Total heat transfer coefficient. Red triangles curve: s-polarization evanescent waves contribution. Blue square curve: p-polarization evanescent waves contribution. The temperature is $T=300 \mathrm{~K}$.

with the free carriers as discussed in $\operatorname{refs}^{3,5,9,12,20}{ }^{\prime \prime}$, To support this assumption, we plot $A=\frac{4 \operatorname{Im}\left[r_{p}\right]^{2} e^{-2 \gamma^{\prime \prime l} l}}{\left|1-r_{s}^{2} e^{-2 \gamma^{\prime \prime l} l}\right|^{2}}$ in the $(\omega, \kappa)$ domain in Fig.2.

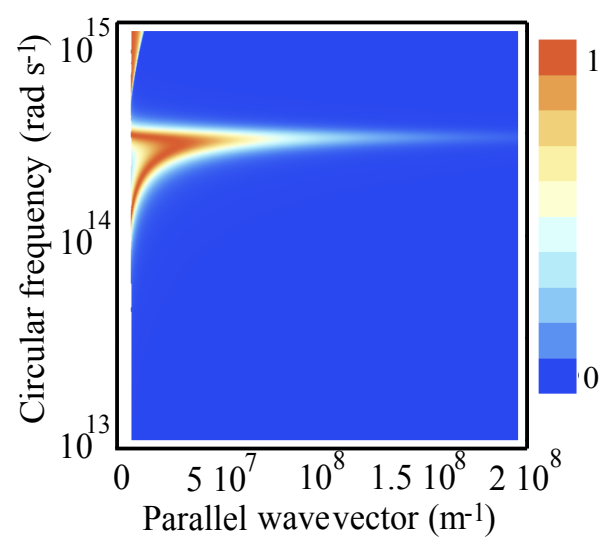

FIG. 2: Colors are available on-line. Ratio $A$ in the plane $(\omega, \kappa)$. Doping concentration is $N_{e}=10^{20} \mathrm{~cm}^{-3}$. The two lines result from the coupling of surface-plasmon polaritons (SPP) on both interfaces. The lines coincide with the dispersion relation of the SPP for the two interfaces system. The gap is $d=10 \mathrm{~nm}$ and the temperature is $T=300 \mathrm{~K}$.

The leading contribution to the heat transfer is confined along two lines. Their shape is given by the dispersion relation of the surface plasmon polariton in the twointerfaces geometry. This behaviour is similar to polar materials which support surface phonon-polariton. The ratio $A$ tends to zero for wave-vectors larger than $1 / d$ because in that case, the surface plasmon on both interfaces no longer overlap. A quantitative estimate of the contribution of the coupled Surface Plasmon Polariton $h_{p}^{s p p}(d, T)$ can be derived by noting that the parallel wavevector satisfies $\kappa \gg \omega / c$. Hence, the Fresnel coefficients become independent of the parallel wave vector $\kappa$ : $\tilde{r}_{p}=\frac{\varepsilon(\omega)-1}{\varepsilon(\omega)+1}$ so that the integration over $\kappa$ can be performed with the help of the second-order polylogarithm function ${ }^{22}$ :

$$
\begin{array}{r}
h_{p}^{s p p}(u, d, T)=\frac{3}{2 \pi^{3}} \frac{g_{0}}{d^{2}} \frac{\operatorname{Im}\left(\tilde{r}_{31}^{p}\right) \operatorname{Im}\left(\tilde{r}_{32}^{p}\right)}{\operatorname{Im}\left(\tilde{r}_{31}^{p} \tilde{r}_{32}^{p}\right)} \times \\
\operatorname{Im}\left[\operatorname{Li} i_{2}\left(\tilde{r}_{31}^{p} \tilde{r}_{32}^{p}\right)\right] u^{2} \frac{e^{u}}{\left(e^{u}-1\right)^{2}},
\end{array}
$$

This closed-form expression (eq.4) is general and also applies when the doping is different for the two plates. It accounts for the temperature dependence and the doping dependence. In order to compare it with the exact numerical result of Eq.(3), we define the thermal conductance $\delta G\left(N_{e}, T\right)$ as:

$$
h_{p}^{s p p}(d, T)=\frac{\delta G\left(N_{e}, T\right)}{d^{2}} .
$$

$\delta G\left(N_{e}, T\right)$ is compared with the exact numerical result in Fig.3. In contrast with previous approximate formulas ${ }^{5,11,20}$, the new asymptotic expression describes accurately the surface mode contribution for a large range of doping levels $10^{16} \leq N_{e} \leq 10^{21} \mathrm{~cm}^{-3}$ and temperatures $100 \leq T \leq 1000$.
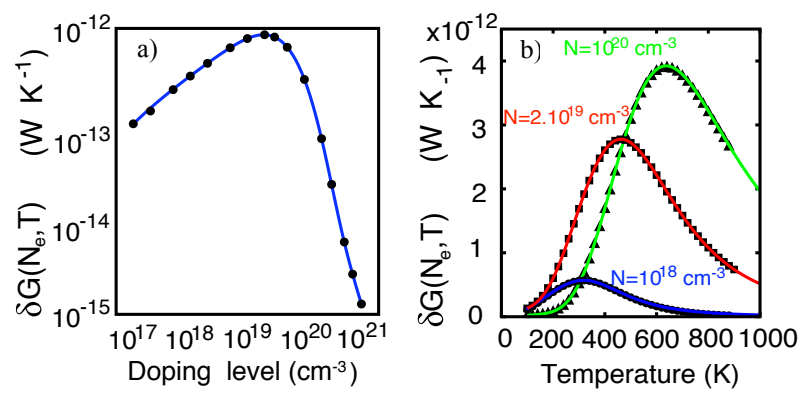

FIG. 3: Colors are available on-line. Comparison between the closed-from expression (plain line) of $\delta G\left(N_{e}, T\right)$ and the exact numerical integration (symbols). a) Dependance on doping at $T=300 K$. b) Dependance on temperature for three doping concentrations.

Changing the doping level can increase the SPP contribution by several orders of magnitude as seen in Fig.3a. Fig.3-b shows that whatever the doping level is, a maximum is found for a precise temperature. However tuning the temperature changes $\delta G\left(N_{e}, T\right)$ by less than one order of magnitude. In order to maximize the nearfield radiative heat transfer, we plot $\delta G\left(N_{e}, T\right)$ versus $N_{e}$ and $T$ (Fig.4). It is seen that there is a maximum for $N_{e} \simeq 10^{20} \mathrm{~cm}^{-3}$ and $T \simeq 600 \mathrm{~K}$ corresponding to $3.910^{-12} \mathrm{WK}^{-1}$. This has the same order of magnitude than $\mathrm{SiC}$ at $300 \mathrm{~K}\left(9.110^{-13} \mathrm{WK}^{-1}\right)$ or silica at $300 \mathrm{~K}$ $\left(2.810^{-12} \mathrm{WK}^{-1}\right)$.

Finally, we note that the heat transfer coefficient can be viewed as the quantum of thermal conductance $g_{0}$ 


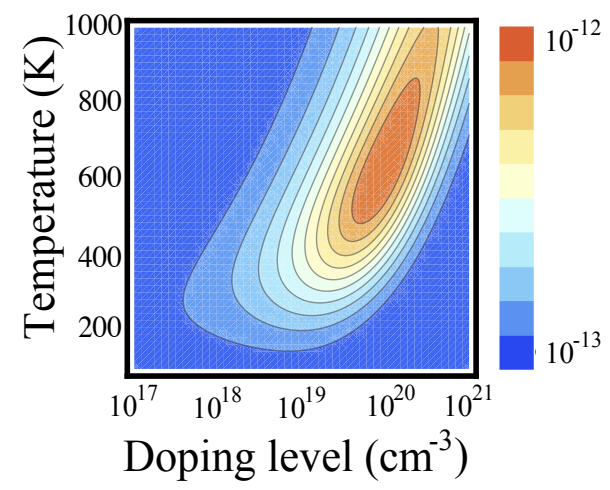

FIG. 4: Colors are available on-line. Evolution of $\delta G\left(N_{e}, T\right)$ with temperature and doping level. $\delta G\left(N_{e}, T\right)$ is maximal for temperature $T \simeq 600 \mathrm{~K}$ and doping level $N_{e}=10^{20} \mathrm{~cm}^{-3}$. times the number of modes per unit area. As expected the number of modes is proportional to $\lambda_{T}^{-2}$ in the far field regime (eq.2). The $1 / d^{2}$ dependance of the radiative heat transfer coefficient in the near-field regime (eq.4) comes from the number of modes per unit area contributing to the heat transfer.

In summary, we have derived an asymptotic closedform expression for the nanoscale heat transfer coefficient mediated by surface plasmons. We showed that the heat transfer coefficient for doped silicon depends on doping and temperature. It has a maximum that can be as large as for polar materials.

The authors acknowledge the support of Agence $\mathrm{Na}$ tionale de la Recherche through Monaco projects and Leti-Carnot Institute.
1 E. G. Cravalho, C. L. Tien and R. P. Caren, J. Heat Transfer 89, 351 (1967).

2 S. M. Rytov, Y. A. Kravtsov, and V. I. Tatarskii, Principles of Statistical Radiophysics, Vol. 3 (Springer, New York, 1989).

3 D. Polder and M. van Hove, Phys. Rev. B 4, 3303 (1971).

${ }^{4}$ M.L. Levin, V. G. Polevoy and S. M. Rytov, Zh. Eksp. Teor. Fiz. 79, 2087 (1980) [Sov. Phys. JETP 52, 1054 (1980)].

5 J. J. Loomis and H. J. Maris, Phys. Rev. B 50, 18517 (1994).

6 J. B. Pendry, J. Phys.: Condens. 11, 6621-6633 (1999).

7 A.I. Volokitin and B.N.J. Persson, Phys. Rev. B 63, 205404 (2001).

8 A.I. Volokitin and B.N.J. Persson, Rev. Mod. Phys., 79, 1291 (2007).

9 K. Joulain, J.-P. Mulet, F. Marquier, R. Carminati, J.-J. Greffet, Surf. Sci. Rep. 57, 59-112 (2005).

10 E. A. Vinogradov, I. A. Dorofeyev, Physics-Uspekhi 52, 425 (2009).

11 J-P. Mulet, K. Joulain, R. Carminati and J-J. Greffet, Microscale Thermophysical Engineering 6, 209 (2002).

12 J.P. Mulet, K. Joulain, R. Carminati, J.J. Greffet,
Appl.Phys.Lett. 78, 2931 (2001).

13 C.M. Hargreaves, Phys. Lett. 30A, 491 (1969).

14 G.A. Domoto, R.F. Boehm and C.L. Tien, J. Heat Transfer 92, 412 (1970).

15 A. Kittel, W. Müller-Hirsch, J. Parisi, S.A. Biehs, D. Reddig, and M. Holthaus, Phys. Rev. Lett. 95, 224301 (2005).

16 U. F. Wischnach, J. Welker, M. Munzel and A. Kittel, Rev. Scientific Instrument 79, 073708 (2008).

17 A. Narayanaswamy and G. Chen, Phys. Rev. B 78, 115303 (2008).

18 S. Shen, A. Narayanaswamy, G. Chen, Nano Lett. doi: 10.1021/nl901208v (2009).

19 E. Rousseau, A. Siria, G. Jourdan, S. Volz, F. Comin, J. Chevrier and J-J. Greffet, Nature Photonics DOI 0.1038/NPHOTON.2009.144 (2009)

20 C.J. Fu and Z.M. Zhang, Int. Jour. Heat Mass Transfer 49, 1703 (2006).

21 E.D. Palik, Handbook of optical constants of solids, Volumes 1-4, Academic Press, New York

22 M. Abramowitz and I. A. Stegun (ed.) Handbook of Mathematical Functions, National Bureau of Standards, 1964; reprinted Dover Publications, 1965. 